\title{
Responses of Ammonia-Oxidizing Archaea to Three Biofertilizers in Organic Soybean Fields
}

\author{
Yazhen Yang ${ }^{1,2}$, Mingke Fang ${ }^{2}$, Meiyan $\mathrm{Wu}^{1}, \mathrm{Jun}_{\mathrm{Hou}}{ }^{1}$ and Jianqiang $\mathrm{Zhu}^{1 *}$ \\ ${ }^{1}$ College of Agriculture, Yangtze University, Jingzhou 434025, China \\ ${ }^{2}$ College of Life Sciences, Yangtze University, Jingzhou 434025, China \\ *For correspondence: 371995966@yangtzeu.edu.cn \\ Received 28 July 2020; Accepted 29 August 2020; Published 10 December 2020
}

\begin{abstract}
Ammonia-oxidizing archaea (AOA) are one of the main regulators of ammoxidation, which is the primary rate-limiting step of nitrification. However, the biofertilizers' effects on soil AOA are not presently well-understood. Therefore, soil samples treated with three biofertilizers viz., nitrogen-fixing bacterium, phosphate-solubilizing bacterium and Piriformospora indica, both individually and mixed (MI) were collected, in order to explore the variation in the AOA composition and diversity using high-throughput sequencing. The results revealed that the available nitrogen content in soil treated with nitrogen-fixing bacterium and MI were significantly higher than that in soil treated with sterile water. In addition, the MI treatment significantly increased the diversity of AOA in soil. The composition of AOA was not different among the treatments at phylum, class, order, or family level. However, this was somewhat different at the genus and species level. Nitrososphaera was dominant at the genus level among the different soil samples. The structural similarity of AOA communities was higher in the three soil samples treated with Piriformospora indica, phosphate-solubilizing bacterium and sterile water, while the communities in soil treated with nitrogen-fixing bacterium and the mixture of nitrogen-fixing bacterium (MI) were different from that in sterile water. The partial least squares discriminant analysis revealed that the classification models for $P$. indica, sterile water and phosphate-solubilizing bacterium performed well. The correlation network analysis demonstrated that there was a positive correlation between Candidatus, Nitrosotalea and Nitrosopumilus. The phylogenetic analysis of the AOA community revealed that ammonia-oxidizing archaea mostly belonged to Thaumarchaeota, followed by Crenarchaeota. In addition, there were some unknown archaea. (C) 2021 Friends Science Publishers
\end{abstract}

Keywords: Ammonia-oxidizing archaea; Biofertilizers; Biodiversity; High-throughput sequencing; Organic soybean soil

\section{Introduction}

Nitrification is a crucial aerobic process in the nitrogen cycle on a global scale, which can reduce nitrate leaching and $\mathrm{N}_{2} \mathrm{O}$ release in the soil (Gao et al. 2018a). It is known that the critical rate-limiting step of nitrification is ammonia oxidization through ammonia-oxidizing bacteria (AOB) and ammonia-oxidizing archaea (AOA). For a long time, it has been considered that the former is the only participant and the main promoter for ammonia oxidation. The dominant position of AOB was removed when the first related archaea strain, Nitrosopumilus martimus SCM1, was isolated from seawater at the Seattle Aquarium in 2005. Since then, ammonia-oxidizing archaea have become a research hotspot, since these are new nitrogen-cycling microorganisms (Könneke et al. 2005). It has also been increasingly realized that ammonia-oxidizing archaea are important functional archaea, because these promote ammonia oxidation in the nitrogen cycle in natural environments (Hatzenpichler 2012).
Due to its unique physiology, the archaea group that oxidizes ammonia was proposed as a new phylum, Thaumarchaeota (Wu et al. 2019). It widely exists in soils, hot springs, marine sediments, fresh water and other environments, and is even more abundant and more sensitive to environmental changes, when compared to $\mathrm{AOB}$, in soil (Zhou et al. 2016). AOA abundance and communities are often correlated to soil properties in agroecosystems. Some studies have shown that $\mathrm{pH}$ is the dominant factor that promotes the variation of AOA community ( $\mathrm{Hu}$ et al. 2014; Li et al. 2015). Substrate availability, such as the amount of ammonium, is also a key driver for the richness of AOA species (Zhang et al. 2015; Norman and Barrett 2016). Soil properties, including soil moisture, temperature, and manure use, may also influence the activity of AOA (Liu et al. 2015a; Rudisill et al. 2016; Zhang et al. 2017). Some studies have also verified that AOA are crucial ammonia-oxidizing prokaryotes under various conditions on land, indicating that it can play more important roles in nitrification, when 
compared to the bacterial counterparts (Prosser and Nicol 2012; Zhang et al. 2012; Liu et al. 2015b). Indeed, some debate remains on this opinion.

Fertilization is an important agricultural practice that is often employed to improve soil nutrition and plant yield. However, it also intensively impacts the AOA community in soil (Liu et al. 2019). Some studies showed that long-term field fertilization can prominently change the community structure of AOB, rather than that of archaea, in paddy soil, and that the former were more susceptible to $\mathrm{N}$, when compared to the latter, in a temperate steppe, indicating that AOB are more important (Wu et al. 2011; Chen et al. 2013). However, some studies revealed that the long-term application of milk vetch raised the abundance of AOA and AOB in red paddy soil. Furthermore, the long-term application of green manures has had larger effects on the AOA community, when compared to the AOB community, in red paddy soil (Aalto et al. 2018; Gao et al. 2018a, b). In addition, an affinity informs the environmental cooperation between them (Straka et al. 2019). These results suggest that AOA plays complex roles in different environments.

Biofertilizers are playing important roles in China's high-efficiency chemical fertilization policy. Nitrogen-fixing bacterium, phosphate-solubilizing bacterium and Piriformospora indica are the three kinds of important biofertilizeres, and these have received attention from researchers (Jayathilake et al. 2006; Aggani 2013). However, studies on AOA communities are still rare for soils treated with biofertilizeres. In order to determine the effects of these three biofertilizers on its communities, a study on the response of AOA to the three biofertilizers was performed in soils in the Swan Island organic field. These results would provide a theoretical basis for understanding the biodiversity in soils, and reveal the mechanism for accelerating the nitrogen cycle with different biofertilizers.

\section{Materials and Methods}

\section{Site description and treatment}

The test field was located on Swan Island in Shishou City, Hubei Province, China, where a cotton-soybean rotation was in place. As the old waterway of the Yangtze River, the location was unique due to its water erosion patterns and mud sediments. Five treatments were set for the experiment: (1) sterile $\mathrm{H}_{2} \mathrm{O}, \mathrm{CK}$; (2) nitrogen-fixing bacteria, NF; (3) phosphate-solubilizing bacteria, PS; (4) P. indica, PI; (5) the mixture of the three strains. MI. NF and PS were provided by our research group, and PI was presented by Professor Kaiwen Ye from Taiwan University. The above bacterial agents were diluted with sterile $\mathrm{H}_{2} \mathrm{O}$ before use. The effective number of viable bacteria in the diluted liquid bacteria agent was $5.0 \times 10^{8} \mathrm{~mL}^{-1}$. The bacterial agents were sprayed at a distance of $3 \mathrm{~cm}$ from the seedlings at 25 days after sowing. Three replicates were performed for each treatment. The area of each replicate was $3 \times 10 \mathrm{~m}^{2}$ in the field experiment, and $900 \mathrm{~mL}$ of bacterial solution was applied for each replicate. The distance between the blocks was $0.5 \mathrm{~m}$. The soybean sowing quantity was $112 \mathrm{~kg} \mathrm{ha}^{-1}$. The experimental blocks were randomly arranged, and the methods of field management were based on local agricultural practices. The soil samples were collected after the soybean was harvested. These soil samples were collected in a plum blossom shape in each block. The 0-30 $\mathrm{cm}$ depth soils were collected after the removal of stones and crop residues, and placed in a $-20^{\circ} \mathrm{C}$ soil refrigerator for conservation. The potted experiments were carried out at the Agricultural Environment and Ecological Engineering Laboratory in Yangtze University. The used pot was $25 \mathrm{~cm}$ in upper diameter, $20 \mathrm{~cm}$ in bottom diameter and $30 \mathrm{~cm}$ in height. The soil of the cultivated layer $(0-30 \mathrm{~cm})$ in test field was placed into the pot. The soil physical and chemical properties were as follows: organic matter content was $16.57 \mathrm{~g} \mathrm{~kg}^{-1}$, total nitrogen amount was $1.16 \mathrm{~g} \mathrm{~kg}^{-1}$, alkalihydrolyzed nitrogen amount was $53.62 \mathrm{mg} \mathrm{kg}^{-1}$, and soil $\mathrm{pH}$ was 7.65. The experimental treatment was the same as the field experiment, and the amount of bacterial agent was 10 $\mathrm{mL}$ per pot. When cotyledon was fully developed, two seedlings were preserved per pot. The field experiment and pot experiment were sown on April 20, 2019. The samples were taken at the maturity stage, and the seeds were tested for yield (Fehr et al. 1971). The number and dry weight of the nodule under the three biofertilizer treatments in the pot experiment were measured according to Yan and Han report (Yan and Han 2014).

\section{Soil available nitrogen content}

The available nitrogen (AN) content in soil from the field experiments was estimated using the Subbiah and Asija method (Subbiah and Asija 1956). A 2 g soil sample was placed in the outer chamber of a clean diffusion dish. The diffusion dish was gently rotated to make the soil sample distribution even. A $2 \mathrm{~mL}$ indicator solution of $\mathrm{H}_{3} \mathrm{BO}_{3}$ was placed into the inner chamber of the diffusion dish, and basic glycerin was smeared on the edge of the outer chamber and covered with ground glass. Then, $10 \mathrm{~mL}$ of $\mathrm{NaOH}$ solution $(1 M)$ was added through a hole on one side of the ground glass, and the diffusion dish was covered tightly and gently rotated to cover all soil samples with lye. Afterwards, the diffusion dish was carefully placed in a constant temperature incubator $\left(40 \pm 1^{\circ} \mathrm{C}\right)$. After alkaline diffusion for $24 \mathrm{~h}$, the $\mathrm{NH}_{3}$ in the inner chamber was titrated with $0.025 \mathrm{M} \mathrm{H}_{2} \mathrm{SO}_{4}$ solution. The process was repeated for three times for each sample.

\section{Gene amplification and sequencing of AOA}

The total DNA of $0.5 \mathrm{~g}$ of soil sample obtained from each field treatment was isolated using a MoBio PowerSoil ${ }^{\mathrm{TM}}$ DNA Isolation Kit (MoBio Laboratories, Carlsbad, CA, USA). Three repeats were performed for each treatment. A 
Nanodrop ND-2000 UV-VIS spectrophotometer (NanoDrop Technologies, Wilmington, DE, USA) and $0.8 \%$ agarose gel electrophoresis were employed to determine the DNA quantity and quality, respectively. The high-quality DNA was conserved at $-80^{\circ} \mathrm{C}$ in an icebox for further analysis. The highly variable V4 region of the bacterial $16 \mathrm{~S}$ rRNA gene (about $250 \mathrm{bp}$ ) was used for sequencing, and the primer $520 \mathrm{~F}$ and $802 \mathrm{R}$ were selected to use PCR amplification. The PCR conditions were based on those described by Francis et al. (2005). The paired-end sequencing of bacterial amplicons was conducted on the Illumina MiSeq sequencer at Personal Biotechnology Co., Ltd. (Shanghai, China).

\section{Quantification of bacterial abundance}

The sequencing data were analyzed using QIIME (v1.8.0) pipeline (Caporaso et al. 2010). The raw reads were classified into the respective samples, according to the barcodes. After the low-quality sequences (sequence length of $<150 \mathrm{bp}$, sequence average Phred score of $<20$, sequences with ambiguous bases and sequences with mononucleotide repeats of $>8 \mathrm{bp}$ ) were filtered out (Chen and Jiang 2014), the clean reads were obtained. The pairedend reads were assembled to use FLASH (Magoc and Salzberg 2011). Rarefaction curves were used to estimate the depth of the sequencing library of each soil type. After chimera detection, the sequences were clustered into operational taxonomic units (OTUs) at 97\% sequence identity utilized UCLUST (Edgar 2010). A representative sequence was chose from each OTU using default parameters. The OTU classification was performed through BLAST, searching the representative sequences set against the Greengenes database for the best hit (DeSantis et al. 2006). Then, each OTU abundance in each sample was analyzed.

\section{Bioinformatics analysis}

The QIIME and R packages (v. 3.2.0) were primarily used to analyze the sequence data. The alpha diversity indices (Chao1 richness estimator, ACE metric, and Shannon diversity index) were analyzed. The OTU richness and evenness among the samples were compared using the ranked abundance curves. Principal component analysis (PCA) was conducted in terms of the genus-level compositional profiles. The differences in microbial structure among the samples were assessed using the $\mathrm{R}$ package "vegan". A Venn diagram was generated to visualize the shared and unique OTUs among samples using the R package "VennDiagram". The taxa abundances were compared among the samples using Metastats. Partial least squares discriminant analysis (PLS-DA) was also introduced to determine the microbiota changes among the samples using the R package "mixOmics" (Chen et al. 2011). The Spearman's rank correlations between the predominant taxa were calculated in order to perform cooccurrence analysis. Correlations $(|\mathrm{RHO}|>0.6$ and $P<0.01)$ were visualized as co-occurrence networks using Cytoscape (Shannon et al. 2003). In addition, the microbial functions were analyzed using PICRUSt (Langille et al. 2013).

\section{Statistical analysis}

S.A.S. 8.1 was used to perform the one-way analysis of variance (ANOVA) and correlation analysis, and Duncan's test was used to evaluate the significance $(P<0.05)$ in the ANOVA.

\section{Results}

Effects of three biofertilizers on the content of available nitrogen

Compared to $\mathrm{CK}$, the content of available nitrogen (AN) varied to some extent in soils treated with the three biofertilizers (Table 1). The highest AN content (106.98 mg $\mathrm{kg}^{-1}$ and $69.81 \mathrm{mg} \mathrm{kg}{ }^{-1}$ ) occurred in soils treated with nitrogen-fixing bacterium during the pot experiment and field experiment, respectively, while the AN content was the lowest $\left(61.76 \mathrm{mg} \mathrm{kg}^{-1}\right.$ and $\left.52.84 \mathrm{mg} \mathrm{kg}^{-1}\right)$ in soils treated with phosphate-solubilizing bacterium during the pot experiment and field experiment, respectively. The content of $\mathrm{AN}$ in soils treated with the nitrogen-fixing bacterium significantly increased by $50.31 \%$ in the pot experiment and $29.49 \%$ in the field experiment, when compared to that in CK $(P<0.05)$. The AN content in soils treated with the solution of phosphate-solubilizing bacterium, $P$. indica, or the mixture of the three biofertilizers increased in the pot experiment and field experiment, when compared to CK, but the differences did not reach a significant level.

\section{Changes in soybean yield and nodules under different biofertilizer treatments}

The different biofertilizer treatments had certain effects on soybean yield and its constituent factors (Table 2). The application of MI, NF and PI could significantly increase the soy yield and its components $(P<0.05)$. Among these, MI had the most obvious effect on the increase in soybean yield under the pot or field experiment. Compared with CK, the soy yield increased by $15.00 \%$ in the pot experiment and increased by $8.57 \%$ in the field experiment under MI treatment. However, the effects of these different treatments on plant height were not significant. The root nodule number varied between 82-116 under the different biofertilizer treatments. The number of soybean root nodules under NF treatment increased most significantly $(P$ $<0.05)$, followed by the MI treatment and PI treatment. The effects of the different biofertilizer treatments on root nodule weight were similar to the number of nodules $(P<0.05)$. The nodule weights/sizes were, as follows: NF $>$ MI $>$ PI $>$ 
Table 1: The content of AN in soils treated with three biofertilizers

\begin{tabular}{lll}
\hline Treatment & Pot experiment $\left(\mathrm{mg} \mathrm{kg}^{-1}\right)$ & Field experiment $\left(\mathrm{mg} \mathrm{kg}^{-1}\right)$ \\
\hline CK & $53.43 \pm 0.11 \mathrm{bc}$ & $53.91 \pm 0.15 \mathrm{bc}$ \\
NF & $106.98 \pm 0.32 \mathrm{a}$ & $69.81 \pm 0.36 \mathrm{a}$ \\
PS & $61.76 \pm 0.52 \mathrm{bc}$ & $52.84 \pm 0.64 \mathrm{bc}$ \\
PI & $66.52 \pm 0.28 \mathrm{bc}$ & $57.24 \pm 0.45 \mathrm{ab}$ \\
MI & $69.08 \pm 0.13 \mathrm{~b}$ & $61.08 \pm 0.22 \mathrm{~b}$ \\
\hline Note: 1$)$ CK: sterile water; 2$)$ NF: nitrogen-fixing bacterium; 3) PS: phosphate solubilizing bacterium; 4) PI: Piriformospora indica; 5) MI: a mixture of the three strains. Data were \\
displayed in the form of mean \pm SD $(\mathrm{n}=3$;); t tests were used to detect the difference in the content of AN in soils treated with three biofertilizers
\end{tabular}

Table 2: Changes of soybean yield under biofertilizer treatment

\begin{tabular}{|c|c|c|c|c|c|c|}
\hline \multirow[t]{2}{*}{ Treatment } & \multicolumn{5}{|c|}{ Pot experiment (per plant) } & \multirow{2}{*}{$\begin{array}{l}\text { Field experiment } \\
\text { Yield }(\mathrm{kg} / \mathrm{ha})\end{array}$} \\
\hline & Height $(\mathrm{cm})$ & Pod number & Pod weight (g) & Pod Seed number & Yield $(\mathrm{g})$ & \\
\hline CK & $46.37 \pm 0.92 \mathrm{a}$ & $7.99 \pm 0.08 c$ & $1.87 \pm 0.07 \mathrm{~b}$ & $28.26 \pm 0.24 c$ & $5.33 \pm 0.05 b$ & $2100 \pm 10.53 b$ \\
\hline $\mathrm{NF}$ & $46.58 \pm 1.42 \mathrm{a}$ & $11.46 \pm 0.05 \mathrm{a}$ & $2.12 \pm 0.51 \mathrm{a}$ & $36.30 \pm 0.25 \mathrm{a}$ & $6.08 \pm 0.03 \mathrm{a}$ & $2250 \pm 16.88 \mathrm{a}$ \\
\hline PS & $47.16 \pm 1.01 \mathrm{a}$ & $8.02 \pm 0.03 b$ & $1.91 \pm 0.02 \mathrm{ab}$ & $28.34 \pm 0.17 b$ & $5.36 \pm 0.07 \mathrm{~b}$ & $2130 \pm 8.17 \mathrm{~b}$ \\
\hline PI & $46.99 \pm 0.77 \mathrm{a}$ & $11.12 \pm 0.73 \mathrm{a}$ & $2.08 \pm 0.09 \mathrm{a}$ & $35.22 \pm 0.33 \mathrm{a}$ & $5.94 \pm 0.09 \mathrm{a}$ & $2220 \pm 21.08 \mathrm{a}$ \\
\hline MI & $48.32 \pm 0.65 \mathrm{a}$ & $11.16 \pm 0.27 \mathrm{a}$ & $2.22 \pm 0.01 \mathrm{a}$ & $36.25 \pm 0.47 \mathrm{a}$ & $6.13 \pm 0.03 \mathrm{a}$ & $2280 \pm 17.10 \mathrm{a}$ \\
\hline
\end{tabular}

Table 3: Changes of the number and dry weight of soybean root nodule in the pot experiment

\begin{tabular}{lll}
\hline Treatment & Nodule number per plant & Dry weight of nodule per plant \\
\hline CK & $82.29 \mathrm{~d}$ & $188.16 \mathrm{~d}$ \\
$\mathrm{NF}$ & $116.11 \mathrm{a}$ & $298.27 \mathrm{a}$ \\
$\mathrm{PS}$ & $85.56 \mathrm{~d}$ & $188.91 \mathrm{~d}$ \\
PI & $94.78 \mathrm{c}$ & $243.24 \mathrm{c}$ \\
MI & $108.76 \mathrm{~b}$ & $281.67 \mathrm{~b}$ \\
\hline Note: 1$) \mathrm{CK}:$ sterile water; 2) NF: nitrogen-fixing bacterium; 3) PS: phosphate solubilizing bacterium; 4) PI: Piriformospora indica; 5) MI: a mixture of the three strains. Data were
\end{tabular}

PS > CK. Compared to CK, the dry weight of the root nodules in the NF treatment increased by $58.52 \%$. In the MI treatment, the dry weight of the root nodules increased by $49.70 \%$. In the PI treatment, the dry weight of the root nodules increased by $29.27 \%$ (Table 3 ).

\section{Analysis of the valid sequences of ammonia-oxidizing archaea under different treatments}

The paired-end sequencing of DNA fragments from the community of ammonia-oxidizing archaea in soils treated with the three biofertilizers was conducted using the Illumina MiSeq platform. After removing the low-quality sequences, the sequence length of each sample was counted (Table 4). The statistics of length distribution of the sequences revealed that the length was distributed between $300 \mathrm{bp}$ and $400 \mathrm{bp}$ (Fig. 1A). The rarefaction curves revealed that the sequencing number exceeded 40,000, and the whole curve tended to be flat, indicating that the sampling was reasonable, and that the sequencing library of each soil sample reached saturation. Thus, this could truly reflect the ammonia-oxidizing archaea groups in the soil samples (Fig. 1B).

\section{Analysis of OTUs and venn graph of ammonia-oxidizing archaea under different treatments}

After removing the rare OTUs and OTUs with a abundance value lower than $0.001 \%$ of the total sequencing volume of all samples, the remaining OTUs were identified (Table 5).
The number of effective sequences obtained was within the range of 62,681-76,897, and the number of effective OTUs under the NF treatment was the highest under different taxon levels. According to the obtained OTU abundance matrix, the number of OTUs shared among the samples was calculated, and a Venn graph was drawn (Fig. 2A). The results revealed that the shared OUT number among all the treatments was 4,297 . The unique OUT number in the CK, NF, PS, PI and MI treatments were 25, 11, 15, 20 and 57, respectively. The unique OTU number in the MI treatment was higher than that in the CK.

\section{Diversity of ammonia-oxidizing archaea}

In order to compare the ammonia-oxidizing archaea diversity in different soil samples under different biofertilizer treatments, a random resample was initially made for each sample in the matrix of the OTU abundance at the depth level of the minimum sequencing (90\%) to revise the diversity differences generated by the sequencing depth. Then, the QIIME software was used to calculate the chao1, ACE and Shannon diversity indices for each sample (Table 6). There was no difference in the Shannon index in soils among the different bacterial fertilizer treatments and control. Furthermore, the MI treatment had higher Chaol and ACE indices, when compared to the control, and there was a significant difference from the control. The other treatments had lower Chaol and ACE index values, when 
Responses of Ammonia-oxidizing Archaea to Biofertilizers / Intl J Agric Biol, Vol 25, No 1, 2021

Table 4: Analysis of the sequencing amount of ammonia-oxidizing archaea in soils treated with different biofertilizers

\begin{tabular}{ll}
\hline Treatment & Sequencing amount \\
\hline NF1 & 68287 \\
NF2 & 66110 \\
NF3 & 62720 \\
PS1 & 57488 \\
PS2 & 62677 \\
PS3 & 67878 \\
PI1 & 62737 \\
PI2 & 61262 \\
PI3 & 75889 \\
MI1 & 76449 \\
MI2 & 80373 \\
MI3 & 73871 \\
CK1 & 63934 \\
CK2 & 69970 \\
CK3 & 71124 \\
\hline Note: NF: Nitrogen-fixing bacterium; PS: phosphate solubilizing bacterium; PI: Piriformospora indica; MI: their mixture and CK: sterile water
\end{tabular}

Table 5: Analysis of OTU classification

\begin{tabular}{|c|c|c|c|c|c|c|c|c|}
\hline Treatment & Sequence number & Phylum & Class & Order & Family & Genus & Species & Unclassified \\
\hline $\mathrm{NF}$ & 65705.67 & 6016.00 & 5991.33 & 6006.67 & 6006.00 & 3991.00 & 6033.00 & 0 \\
\hline PS & 62681.00 & 5909.33 & 5887.67 & 5899.67 & 5898.33 & 3910.33 & 5925.67 & 0 \\
\hline PI & 66629.33 & 5908.67 & 5869.33 & 5890.33 & 5888.33 & 3852.00 & 5924.00 & 0 \\
\hline CK & 68342.67 & 5931.33 & 5908.33 & 5920.67 & 5919.33 & 3859.67 & 5947.67 & 0 \\
\hline
\end{tabular}

Note: NF: Nitrogen-fixing bacterium; PS: phosphate solubilizing bacterium; PI: Piriformospora indica; MI: their mixtur and CK: sterile water
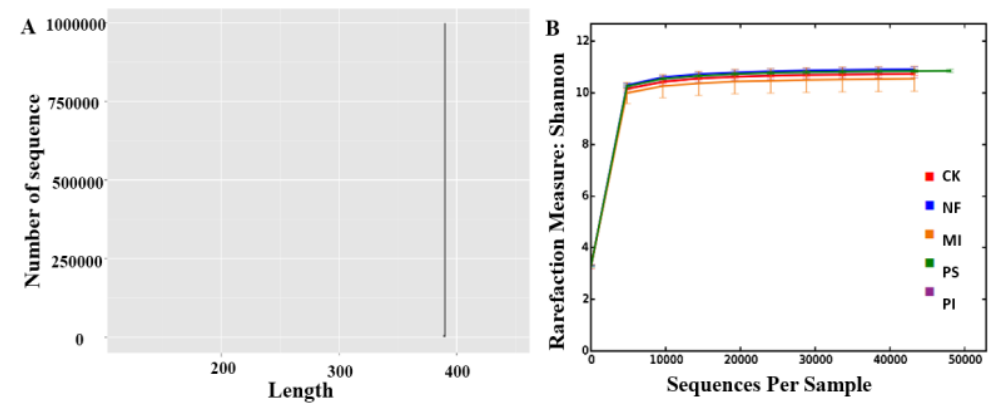

Fig. 1: Assessment of the sequencing data of ammonia-oxidizing archaea in soils treated with different biofertilizers. A. Distribution of sequence length, B. Rarefaction curves. NF: Nitrogen-fixing bacterium; PS: phosphate-solubilizing bacterium; PI: Piriformospora indica; MI: their mixture and CK: sterile water

compared to the control. The PS treatment and PI treatment had significant differences, when compared to the control. In conclusion, MI treatment can significantly increase the AOA diversity in soil.

\section{Taxonomic composition analysis of ammonia-oxidizing archaea}

According to the OTU identification, the specific composition of ammonia-oxidizing archaea from each soil sample can be obtained at different classification levels. However, the composition is not different at the phylum, class, order and family level, while there were some differences at the genus and species level (Table 7). Furthermore, Nitrososphaera was dominant at the genus level in the different soil samples, and Nitrosopumilus, Candidatus Nitrosotalea and Candidatus Nitrosoarchaeum were relatively disadvantaged. In addition, other genera were identified (Fig. 2B). The heat map analysis also revealed that the abundance of Nitrososphaera in samples treated with NF, PI and MI increased, when compared to that in $\mathrm{CK}$ (Fig. 3A).

\section{Analysis of the AOA communities under different treatments}

Principal component analysis (PCA) was used to o assess differences in the AOA composition in soil under different treatments. The results showed the significant clustering of AOA communities (Fig. 3B). PC1 and PC2 explained 99.48 and $0.45 \%$ of the total changes, respectively. NF, PS, PI and MI were obviously distinguished from $\mathrm{CK}$, suggesting that there were significant influences from the different treatments on soil AOA. Furthermore, the community 
Table 6: Diversity of ammonia-oxidizing archaea communities in soil treated with different microbial fertilizers

\begin{tabular}{llll}
\hline Treatment & Shannon & Chao1 & ACE \\
\hline CK & $10.74 \pm 0.04 \mathrm{a}$ & $4930.92 \pm 68.66 \mathrm{~b}$ & $5041.49 \pm 63.19 \mathrm{~b}$ \\
$\mathrm{NF}$ & $10.92 \pm 0.07 \mathrm{a}$ & $4782.62 \pm 37.17 \mathrm{bc}$ & $4818.54 \pm 17.5 \mathrm{bc}$ \\
$\mathrm{OP}$ & $10.85 \pm 0.04 \mathrm{a}$ & $4688.98 \pm 56.54 \mathrm{~cd}$ & $4711.59 \pm 78.49 \mathrm{~cd}$ \\
$\mathrm{PI}$ & $10.75 \pm 0.11 \mathrm{a}$ & $4532.53 \pm 108.77 \mathrm{~cd}$ & $4504.38 \pm 97.6 \mathrm{~d}$ \\
$\mathrm{MI}$ & $10.89 \pm 0.04 \mathrm{a}$ & $5350.93 \pm 52.41 \mathrm{a}$ & $5336.21 \pm 74.83 \mathrm{a}$ \\
\hline Note: 1) CK: & sterile water; 2) NF: nitrogen-fixing bacterium; 3) PS: phosphate \\
solubilizing bacterium; 4) PI: Piriformospora indica; 5) MI: a mixture of the three \\
strains. Data were displayed in the form of mean \pm SD (n = 3); t tests were used to \\
\multicolumn{4}{l}{ detect the difference in the relative indexes in soils treated with three biofertilizers }
\end{tabular}
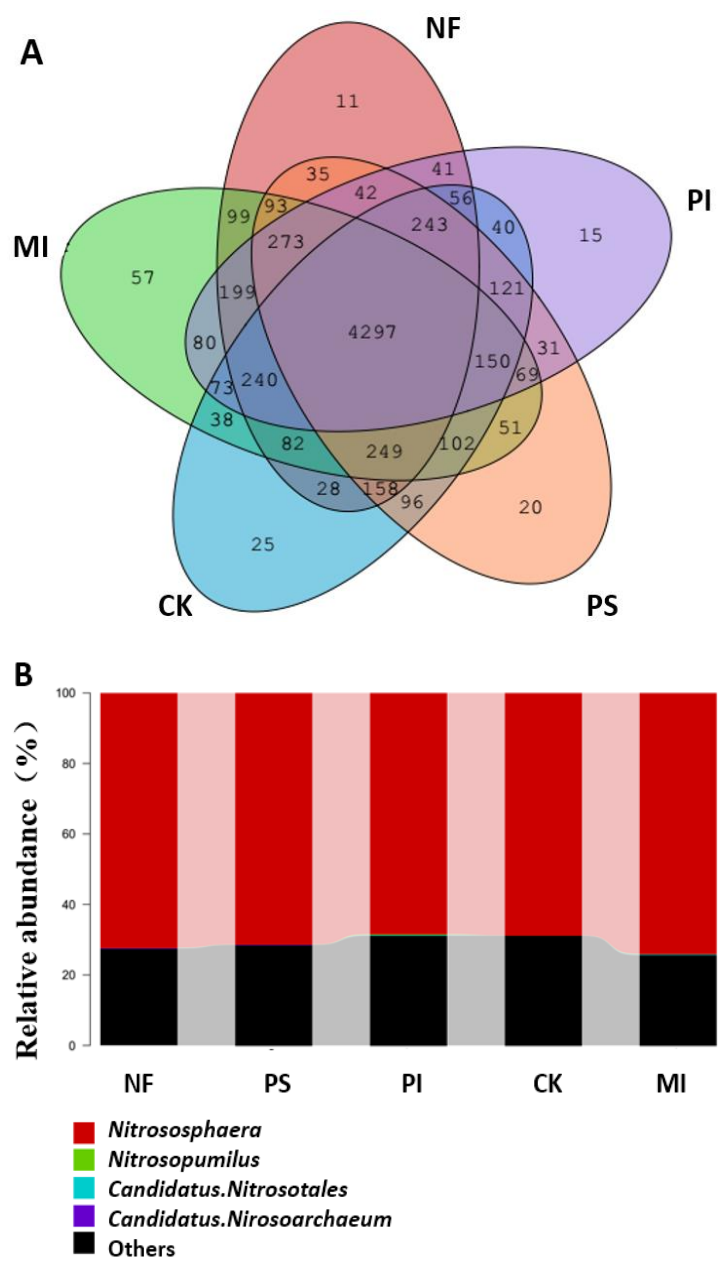

Fig. 2: Analysis of ammonia-oxidizing archaea groups in soils treated with different biofertilizers. A. Venn graph of ammoniaoxidizing archaea groups. B. The relative abundance of ammoniaoxidizing archaea at the genus level. NF: Nitrogen-fixing bacterium; PS: phosphate-solubilizing bacterium; PI: $P$. indica; MI: their mixture and CK: sterile water

difference between PI and CK was the largest, while that between PS and CK was the smallest. The community differences for MI or PS were relatively small, when compared to CK. a PLS-DA discrimination model was established according to the species abundance matrix and sample grouping data for AOA using the R software. The
Table 7: Statistical table of microbial groups at each classification level

\begin{tabular}{lllllll}
\hline Sample & Phylum & Class & Order & Family & Genus & Species \\
\hline NF & 2 & 2 & 3 & 3 & 5 & 13 \\
NF & 2 & 2 & 3 & 3 & 4 & 10 \\
NF & 2 & 2 & 3 & 3 & 4 & 10 \\
PS & 2 & 2 & 3 & 3 & 5 & 11 \\
PS & 2 & 2 & 3 & 3 & 5 & 12 \\
PS & 2 & 2 & 3 & 3 & 5 & 12 \\
PI & 2 & 2 & 3 & 3 & 5 & 12 \\
PI & 2 & 2 & 3 & 3 & 5 & 13 \\
PI & 2 & 2 & 3 & 3 & 5 & 13 \\
MI & 3 & 2 & 3 & 3 & 5 & 17 \\
MI & 2 & 2 & 3 & 3 & 5 & 10 \\
MI & 2 & 2 & 3 & 3 & 5 & 12 \\
CK1 & 2 & 2 & 3 & 3 & 5 & 11 \\
CK2 & 2 & 2 & 3 & 3 & 5 & 11 \\
CK3 & 2 & 2 & 3 & 3 & 5 & 12 \\
\hline
\end{tabular}

Note: NF: Nitrogen-fixing bacterium; PS: phosphate solubilizing bacterium; PI: Piriformospora indica; MI: their mixture and CK: sterile water

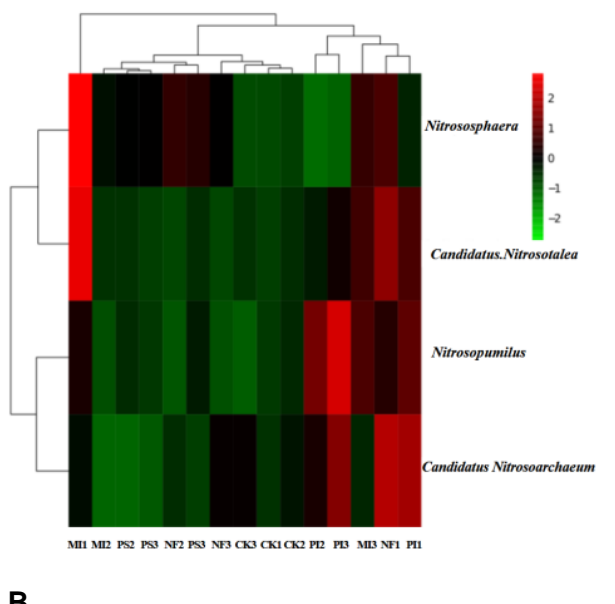

B

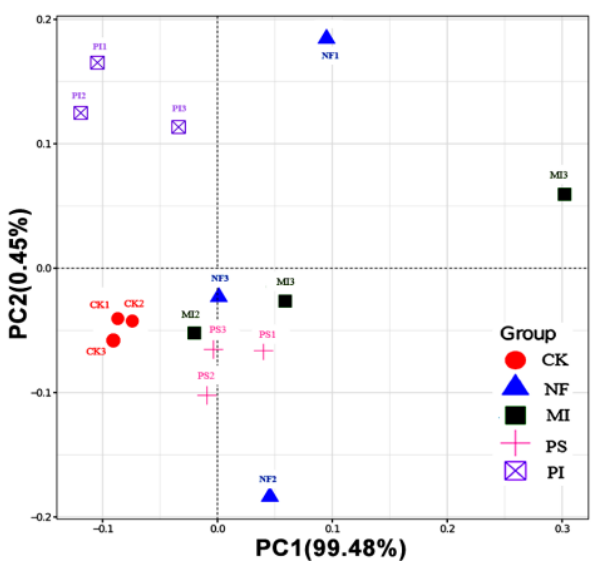

Fig. 3: The relative abundance of ammonia-oxidizing archaea in the soil at the genus level. A. Heat map of the genus level. B. PCA analysis of the structural similarity. NF: Nitrogen-fixing bacterium; PS: phosphate-solubilizing bacterium; PI: Piriformospora indica; MI: their mixture and CK: sterile water

results revealed that the classification models for PI, CK and PS performed well, while those for NF and MI did not 


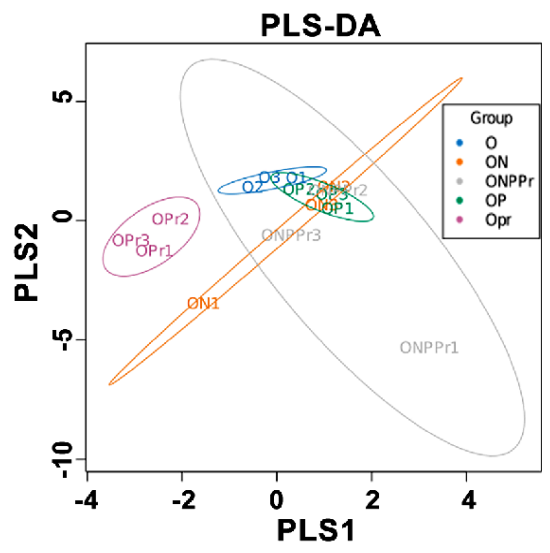

B.

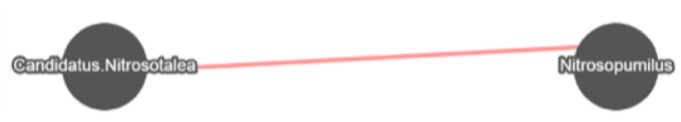

Fig. 4: Comparative analysis of the AOA colony and screening of their key species. A. PLS-DA discriminant analysis. B. The nodes represent the dominant genera and are marked with different colors. The connections between the nodes indicate the correlation between the two genera; a red line indicates a positive correlation, and a green line indicates a negative correlation. The more connections there are through a node, the more associations the genus has with other members of the community. NF (ON): Nitrogen-fixing bacterium; PS (OP): phosphate-solubilizing bacterium; PI (OPr): Piriformospora indica; MI: their mixture (ONPPr) and CK (O): sterile water

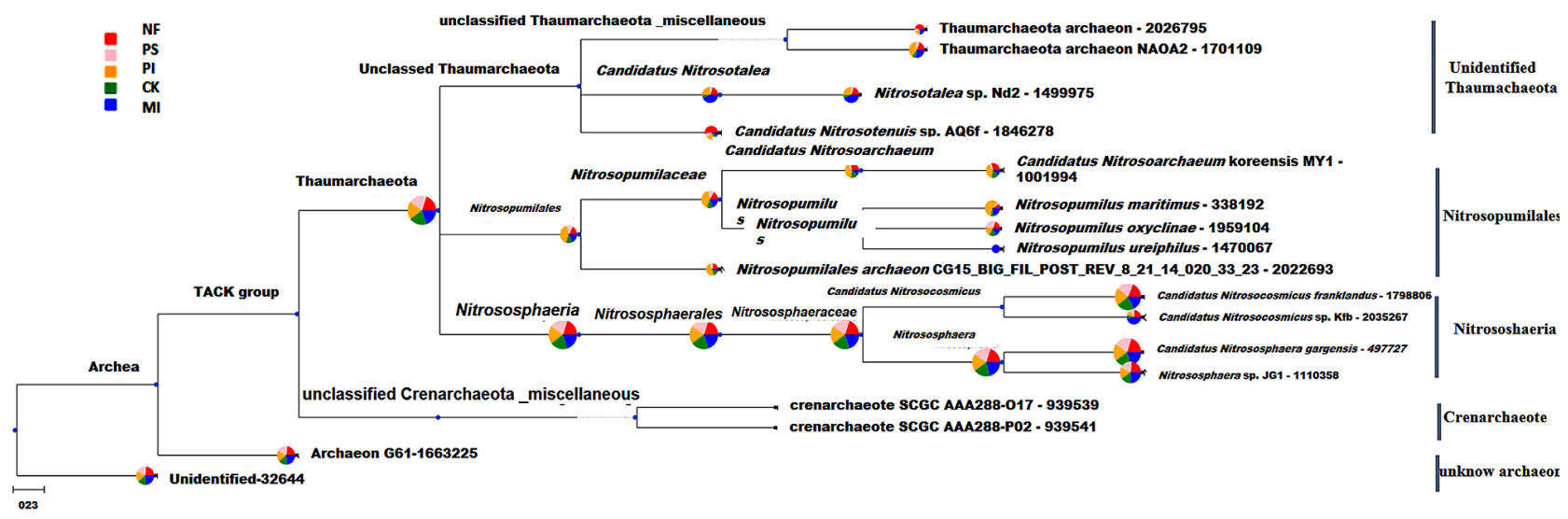

Fig. 5: Map of the evolution and abundance of AOA species. The pie chart of each branch node of the classification showed the abundance of this taxon in each sample. The larger the sector area is, the higher the abundance in the corresponding sample.

perform well (Fig. 4A). Among these, the classification model for PI was the best, and was quite different from CK, suggesting that the community difference between PI and CK is significant.

\section{Analysis of the correlation network among the dominant species of AOA}

In terms of the abundance distribution of OTUs or taxa in the different soil samples, AOA groups that were negatively or positively correlated with each other can be found, and an association network of the dominant AOA groups can be built to analyze the ecological significance of the correlation
(Fig. 4B). In the present study, with the use of the Mothur software, the Spearman's correlation coefficient was calculated between the dominant genera, in which the abundance was within the top 50. The correlation network was constructed for the dominant genus with an abundance of rho of $>0.6$ and a P-value of $<0.01$, and the correlation network was imported into the Cytoscape software for visualization. The results revealed a positive correlation between Candidatus, Nitrosotalea and Nitrosopumilus, which indicating that there was a cooperative relationship between these. However, the correlation networks between the other dominant genera were relatively low. 


\section{Phylogenetic analysis of AOA communities}

In the process of OTU identification, the phylogenetic tree that represented the sequence of OTUs was constructed using the Fast Tree tool. Using the MEGAN software, the OUT abundance and composition data in each soil sample were mapped to the classification hierarchy tree provided by the NCBI Taxonomy. The result revealed that ammoniaoxidizing archaea mostly belonged to Thaumarchaeota (including Nitrosopumilales, Nitrososphaeria and unidentified Thaumarchaeota), followed by Crenarchaeota. In addition, there were some unknown archaea (Fig. 5).

\section{Discussion}

Nitrification is an important part of the process of the nitrogen cycle, and is closely correlated to nitrogen availability (Galloway 2008). Ammonia oxidation is the rate-limiting step of nitrification and plays a crucial role in soil nitrogen cycle. Therefore, as the main contributor to ammonia oxidation, AOA in soil has been a hotspot in agricultural ecosystem research. The ammonia-oxidizing archaea in soils treated with different biological fertilizers at the soybean seedling stage were analyzed in the present study. The results showed that the content of available nitrogen in soil was the highest under the treatment of nitrogen-fixing bacterium in the pot experiment and field experiment, and this followed by MI and $P$. indica. The application of MI, NF and PI can significantly increase the soy yield and its components. The number of soybean root nodules under NF treatment increased most significantly, followed by the MI treatment and PI treatment. The effects of the different biofertilizer treatments on root nodule weight were similar to the number of nodules. These results revealed that the biofertilizer has important effects on the nitrogen fixation capacity of soils (Chakraborty and Tribedi 2019). These promote the uptake and utilization of nitrogen by plants, and ultimately promote the formation and development of seeds. So, do these biofertilizers have an effect on the ammonia-oxidizing archaea in the soil?

In fact, the community composition of AOA in soil is influenced to different extent by the applied fertilization treatments. For example, the long-term chemical fertilization had significant effects on the community composition of AOA in soil (He et al. 2007). However, organic fertilization had little effects on AOA (Wang et al. 2014; Tao et al. 2017). In this study, the changes in ammonia-oxidizing archaea in soil under different bacterial fertilizer treatments were analyzed using high-throughput sequencing technology. The results revealed that the number of unique OTUs in the MI treatment was the highest among all treatments. The Chao1 and ACE indices focus on the analysis of community richness (Chao 1984; Chao and Yang 1993). Compared to the control, the single-bacteria fertilizer treatments had little effect on the Chaol and ACE indices, suggesting the stability of the soil community richness.
However, the mixed bacterial fertilizer greatly influenced the Chao 1 and ACE indices. The effects of all kinds of treatments on the Shannon index were evaluated as community evenness, and the results were similar to those for the Chao1 and ACE indices. These results suggest that mixed biofertilizer might better improve the community richness and evenness, when compared to single-bacteria fertilizers. These differences from the applied fertilization treatments could be closed relative to the different soil conditions and fertilezer varieties.

Archaea are important components of complicated microbiomes in the holobiont environment. These interplay closely with viruses, microorganisms and holobionts, such as plants and animals (Moissl-Eichinger et al. 2018). Therefore, the changes in microbe species in soil must influence archaea, to some extent. These present results revealed the changes in archaea taxa at the genus and species level. Furthermore, Nitrososphaera was dominant at the genus-level in the different soil samples, implying the greater functional importance of the Nitrososphaera group, when compared to other archaea, in the present study. The heatmap analysis also revealed that different bacterial fertilizer treatments may influence the soil microbe species. These results are consistent with the report of Wu et al. (2019). In addition, the correlation network analysis result revealed that Candidatus Nitrosotalea and Nitrosopumilus were positively correlated. Therefore, on the basis of this study, it is very sinificant to analyze the interaction between the dominant archaea group and three bio-bacterial fetilizers in soil.

\section{Conclusion}

Overall, the NF, PI and MI treatments were not only beneficial for increasing the soybean yield, but also the growth and development of soybean root nodules. the MI treatment significantly increased the diversity of AOA in soil. The composition of AOA was only somewhat different at the genus and species level under different biofertizer treatments. In addition, Nitrososphaera was dominant at the genus level among the different soil samples. The communities in soil treated with nitrogen-fixing bacterium and the mixture of nitrogen-fixing bacterium (MI) were different from that in sterile water. These results confirmed that different biofertizers could influencd the diversity of AOA. However, the interactive mechanisms between these biofertilizers and the dominant group of ammonia-oxidizing archaea are not elucidated well at present. Therefore, these research on ammonia-oxidizing archaea in soil needs to be further enhanced in the future in order to lay the foundation for the effective utilization of biofertilizers.

\section{Acknowledgements}

We acknowledge the financial supports of the organic agriculture development program by the Worldwide Fund for Nature and Rare Conservation under Grant No. PO2893 
and No. PO3042.

\section{Author Contributions}

Yazhen Yang and Jianqiang Zhu conceived and designed the experiments; Mingke Fang and Yazhen Yang performed the experiments; Meiyan $\mathrm{Wu}$ and Jun Hou analyzed the data, Yazhen Yang wrote the manuscript. Jianqiang Zhu revised the manuscript. All authors read and approved the final draft.

\section{References}

Aalto SL, J Saarenheimo, A Mikkonen, AJ Rissanen, M Tiirola (2018). Resistant ammonia-oxidizing archaea endure, but adapting ammonia-oxidizing bacteria thrive in boreal lake sediments receiving nutrient - rich effluents. Environ Microbiol 20:3616-3628

Aggani SL (2013). Development of bio-fertilizers and its future perspective. Soil Sci Soc Amer J 2:327-332

Caporaso JG, J Kuczynski, J Stombaugh, K Bittinger, FD Bushman, EK Costello, N Fierer, AG Pena. JK Goodrich, JI Gordon, GA Huttley, ST Kelley, D Knights, JE Koenig, RE Ley, CA Lozupone, D McDonald, BD Muegge, M Pirrung, J Reeder, JR Sevinsky, PJ Tumbaugh, WA Walters, J Widmann, T Yatsunenko, J Zaneveld, R Knight (2010). QIIME allows analysis of high-throughput community sequencing data. Nat Meth 7:335-336

Chakraborty P, P Tribedi (2019). Functional diversity performs a key role in the isolation of nitrogen-fixing and phosphate-solubilizing bacteria from soil. Folia Microbiol 64:1-10

Chao A (1984). Nonparametric estimation of the number of classes in a population. Scand J Stat 11:265-270

Chao A, MCK Yang (1993). Stopping rules and estimation for recapture debugging with unequal failure rates. Biometrika 80:193-201

Chen H, W Jiang (2014). Application of high-throughput sequencing in understanding human oral microbiome related with health and disease. Front Microbiol 5; Article 508

Chen Y, Z Xu, H Hu, Y Hu, Z Hao, Y Jiang, B Chen (2013). Responses of ammonia-oxidizing bacteria and archaea to nitrogen fertilization and precipitation increment in a typical temperate steppe in Inner Mongolia. Appl Soil Ecol 68:36-45

Chen YF, FL Yang, HF Lu, BH Wang, YB Chen, DJ Lei, YZ Wang, BL Zhu, LJ Li (2011). Characterization of Fecal Microbial Communities in Patients with Liver Cirrhosis. Hepatology 54:562-572

DeSantis TZ, P Hugenholtz, N Larsen, M Rojas, EL Brodie, K Keller, T Huber, D Dalevi, P Hu, GL Andersen (2006). Greengenes, a chimera-checked 16S rRNA gene database and workbench compatible with ARB. Appl Environ Microbiol 72:5069-5072

Edgar RC (2010). Search and clustering orders of magnitude faster than BLAST. Bioinformatics 26:2460-2461

Fehr WR, S Canvins, DT Burmood, JS Pennington, JS Pennington (1971). Stage of development descriptions for soybean (Glycine max (L.) Merr.). Crop Sci 11:929-931

Francis CA, KJ Roberts, JM Beman (2005). Ubiquity and diversity of ammonia-oxidizing archaea in water columns and sediments of the ocean. Proc Natl Acad Sci 102:14683-14688

Galloway JN (2008). An earth-system perspective of the global nitrogen cycle. Nature 451:293-296

Gao SJ, DN Chang, CQ Zou, WD Cao, JS Gao, J Huang, JS Bai, NH Zeng, RM Reesg, K Thorup-Kristensen (2018a). Archaea are the predominant and responsive ammonia oxidizing prokaryotes in a red paddy soil receiving green manures. Eur J Soil Biol 88:27-35

Gao S, W Cao, C Zou, J Gao, J Huang, J Bai, F Dou (2018b). Ammoniaoxidizing archaea are more sensitive than ammonia-oxidizing bacteria to long-term application of green manure in red paddy soil. Appl Soil Ecol 124:185-193

Hatzenpichler R (2012). Diversity, physiology, and niche differentiation of ammonia-oxidizing archaea. Appl Environ Microbiol 78:75017510
He J, J Shen, L Zhang, Y Zhu, Y Zheng, M Xu, HJ Di (2007) Quantitative analyses of the abundance and composition of ammonia-oxidizing bacteria and ammonia-oxidizing archaea of a Chinese upland red soil underlong-term fertilization practices. Environ Microbiol 9:2364-2374

Hu B, S Liu, W Wang, L Shen, L Lou, W Liu, G Tian, X Xu, P Zheng (2014). pH dominated niche segregation of ammonia-oxidising microorganisms in Chinese agricultural soils. FEMS Microbiol Ecol 90:290-299

Jayathilake PKS, IP Reddy, D Srihari, KR Reddy (2006). Productivity and soil fertility status as influenced by integrated use of $\mathrm{N}$-fixing biofertilizers, organic manures and inorganic fertilizers in onion. $J$ Agric Sci 2:46-58

Könneke M, AE Bernhard, R José, CB Walker, JB Waterbury, D Stahl (2005). Isolation of an autotrophic ammonia-oxidizing marine archaeon. Nature 437:543-546

Langille MGI, J Zaneveld, JG Caporaso, D McDonald, D Knights, JA Reyes, JC Clemente, DE Burkepile, RLV Thurber, R Knight, RG Beiko, C Huttenhower (2013). Predictive functional profiling of microbial communities using $16 \mathrm{~S}$ rRNA marker gene sequences. Nat Biotechnol 31:814-821

Li H, BS Weng, FYJ Huang, Q Su, XR Yang (2015). pH regulates ammonia-oxidizing bacteria and archaea in paddy soils in Southern China. Appl Microbiol Biotechnol 99:6113-6123

Liu R, H Suter, J He, H Hayden, D Chen (2015a). Influence of temperature and moisture on the relative contributions of heterotrophic and autotrophic nitrification to gross nitrification in an acid cropping soil. J Soil Sedim 15:2304-2309

Liu S, B Hu, Z He, B Zhang, G Tian, P Zheng, F Fang (2015b). Ammoniaoxidizing archaea have better adaptability in oxygenated/hypoxic alternant conditions compared to ammonia-oxidizing bacteria. Appl Soil Ecol 99:8587-8596

Liu T, Z Wang, S Wang, Y Zhao, AL Wright, X Jiang (2019). Responses of ammonia-oxidizers and comammox to different long-term fertilization regimes in a subtropical paddy soil. Eur J Soil Biol 93:1-6

Magoc T, SL Salzberg (2011). FLASH: fast length adjustment of short reads to improve genome assemblies. Bioinformatics 27:2957-2963

Moissl-Eichinger C, M Pausan, J Taffner, G Berg, C Bang, RA Schmitz (2018). Archaea are interactive components of complex microbiomes. Trends Microbiol 26:70-85

Norman JS, JE Barrett (2016). Substrate availability drives spatial patterns in richness of ammonia-oxidizing bacteria and archaea in temperate forest soils. Soil Biol Biochem 94:169-172

Prosser JI, GW Nicol (2012) Archaeal and bacterial ammonia-oxidisers in soil: The quest for niche specialisation and differentiation. Trends Microbiol 20:523-531

Rudisill MA, RF Turco, LA Hoagland (2016). Fertility practices and rhizosphere effects alter ammonia oxidizer community structure and potential nitrification activity in pepper production soils. Appl Soil Ecol 99:70-77

Shannon P, A Markiel, O Ozier, NS Baliga, JT Wang, D Ramage, N Amin, B Schwikowski, T Ideker (2003). Cytoscape: A software environment for integrated models of biomolecular interaction networks. Genome Res 13:2498-2504

Straka L, KA Meinhardt, A Bollmann, DA Stahl, MK Winkler (2019) Affinity informs environmental cooperation between ammoniaoxidizing archaea (AOA) and anaerobic ammonia-oxidizing (Anammox) bacteria. ISME J 13:1431-1439

Subbiah B, G Asija (1956). Alkaline method for determination of mineralizable nitrogen. Curr Sci 25:259-260

Tao R, SA Wakelin, YC Liang, GX Chu (2017) Response of ammoniaoxidizing archaea and bacteria in calcareous soil to mineral and organic fertilizer application and their relative contribution to nitrification. Soil Biol Biochem 14:20-30

Wang Y, G Zhu, L Song, S Wang, C Yin (2014) Manure fertilization alters the population of ammonia-oxidizing bacteria rather than ammoniaoxidizing archaea in a paddy soil. J Basic Microb 54:190-197

Wu RN, H Meng, YF Wang, JD Gu (2019). Functional dominance and community compositions of ammonia-oxidizing archaea in extremely acidic soils of natural forests. Appl Microbiol Biotechnol 103:4229-4240 
Wu Y, L Lu, B Wang, X Lin, J Zhu, Z Cai, X Yan, Z Jia (2011). Long-term field fertilization significantly alters community structure of ammonia-oxidizing bacteria rather than archaea in a paddy soil. Soil Sci Soc Amer J 75:1431-1439

Yan J, XZ Han (2014). Effect of soil inorganic N concentrations on the nodulation, $\mathrm{N}_{2}$ fixation and yield in soybean in a pot experiment. $\mathrm{Sci}$ Agric Sin 47:1929-1938

Zhang LM, HW Hu, JP Shen, JZ He (2012). Ammonia-oxidizing archaea have more important role than ammonia-oxidizing bacteria in ammonia oxidation of strongly acidic soils. ISME J 15:61032-61045
Zhang Q, G Liang, DD Myrold, W Zhou (2017). Variable responses of ammonia oxidizers across soil particle-size fractions affect nitrification in a long-term fertilizer experiment. Soil Biol Biochem 105:25-36

Zhang Y, L Chen, T Dai, R Sun, D Wen (2015). Ammonia manipulates the ammonia-oxidizing archaea and bacteria in the coastal sedimentwater microcosms. Appl Microbiol Biotechnol 99:6481-6491

Zhou XH, YM Li, JP Zhang, B Liu, MY Wang, YW Zhou, ZJ Lin, ZL He (2016). Diversity, abundance and community structure of ammoniaoxidizing archaea and bacteria in riparian sediment of Zhenjiang ancient canal. Ecol Eng 90:447-458 